\title{
Glomerular Number and Perfusion during Normal and Compensatory Renal Growth in the Guinea Pig
}

\author{
ROBERT L. CHEVALIER ${ }^{(19)}$ \\ Department of Pediatrics, University of Virginia, School of Medicine, Charlottesville, Va, U.S.A.
}

\section{Summary}

Changes in glomerular number and perfusion during maturation of the guinea pig were compared with the response to uninephrectomy at birth or 84 days of age. From birth to adulthood, there was a $21 \%$ increase in number of glomeruli identified by the presence of India ink previously injected in vivo. For animals uninephrectomized at birth, a similar increase occurred several wk earlier than in sham-operated littermates; however, uninephrectomy in adulthood resulted in no further increment. Although the number of glomeruli in the left kidney varied by $25 \%$ within each group, the difference in number between right and left kidneys of individual animals averaged only $4 \%$.

Additional glomeruli not identified by India ink were subsequently revealed by application of Wright's stain. These comprised 13-17\% of the glomeruli containing India ink in 1 and 22-day-old sham groups, but only $2 \%$ in 22 -day-old uninephrectomized or adult animals. Approximately $3 / 4$ of all glomeruli indentified by India ink were present in the outer cortex of 22-day-old uninephrectomized and sham-operated guinea pigs; the cortical distribution of Wright-stained glomeruli in the latter group was similar.

Paraffin sections of kidneys revealed scattered glomeruli not containing India ink in 1-day-old and 22-day-old sham guinea pigs, but almost none in 22-day-old uninephrectomized animals. There were no glomeruli in an early stage of formation in any of the sections examined.

It is concluded that during development, an increase in number of glomeruli identified by India ink represents nephrons which are underperfused in the neonate but are completely perfused at maturity. This process is accelerated by uninephrectomy in early development, but is unaffected by uninephrectomy in adulthood, at which time glomerular perfusion is virtually homogeneous.

\section{Speculation}

Postnatal nephrogenesis has been proposed as an adaptive mechanism underlying the rapid increase in glomerular filtration rate of the neonate and enhanced compensatory renal hypertrophy following unilateral nephrectomy in early development. In the guinea pig, a species with renal maturation similar to that in the human, there is no histologic evidence for development of additional nephrons postnatally, but the unique adaptation of renal function to growth requirements may be related to changing glomerular perfusion patterns. Additional studies of glomerular hemodynamics in the newborn with reduced renal mass will be required to elucidate these processes.

The question of whether additional nephrons are formed by the postnatal mammalian kidney has interested anatomists for nearly a century (8) and continues to remain controversial $(4,5,10,12)$. A number of investigators have concluded that in species relatively immature at birth, such as the rat or mouse, nephrogenesis may continue after birth $(1,3,15)$ or following unilateral nephrectomy in early development $(2,3,4,5)$. In contrast, unilateral nephrectomy of the developing rat has been shown by others to result in no increase in nephron number $(10,12)$, and a histologic study by Larsson et al. (12) revealed no developing nephrons after 5 days of age.

In the guinea pig, a species more similar to the human with respect to renal development, an early study showed no change in glomerular number with normal growth (11), but others have found an increase $(3,13)$. Nonetheless, unilateral nephrectomy at 5 or more days of age did not result in additional glomeruli (3).

In view of these conflicting findings, the present study in the guinea pig was designed to examine the sequential effects on nephron number of normal development and of unilateral nephrectomy at birth. Glomeruli were initially identified by the presence of India ink in acid digests of kidneys from animals that had been injected in vivo using a modification of the method of Damadian et al. (7). The number of glomeruli thus detected was found to increase during normal development. The rise occurred at an earlier age in animals uninephrectomized at birth. Because this technique of glomerular counting is dependent on homogeneous perfusion by India ink, preparations were subsequently stained to identify all glomerular and tubular elements. This revealed that in the least mature kidneys, a significant number of glomeruli had not been identified by India ink alone. Furthermore, histologic sections showed no early developing glomeruli. Changes in apparent number of nephrons identified by the method of Damadian et al. may therefore relate to the recruitment of underperfused glomeruli rather than to de novo nephron formation.

\section{MATERIALS AND METHODS}

Studies were performed in 60 male and female guinea pigs weighing $82-860 \mathrm{~g}$. In order to determine the number of glomeruli immediately after birth, six animals were sacrificed within the first day of life. In a second group of 44 animals, right nephrectomy (N) or sham operation (S) was performed under ether anesthesia within the first $36 \mathrm{~h}$ of life by means of a flank incision. The wound was closed, the animal was allowed to recover and was returned to its mother. Animals in each litter were evenly divided into $\mathrm{N}$ or $\mathrm{S}$ groups. These guinea pigs were sacrificed at 10,22 , or 105 days of age to compare the effects of early uninephrectomy with normal growth. In a third group of 10 animals, $N$ or $\mathrm{S}$ operation was performed at 84 days of age. These animals were sacrificed at 105 days, to evaluate the effect of uninephrectomy in adulthood.

In 1- and 105-day-old groups, the animal was subjected to light ether anesthesia and a $5 \mathrm{~mm}$ incision was made in the neck for exposure of a carotid artery, which was cannulated with PE50 polyethylene tubing. India ink (Higgins No. 4465, Newark, NJ), 0.5-2.0 ml, was then slowly infused through the catheter. After 35 min, the animal was killed with an injection of sodium pentobarbital, and the kidney was immediately removed, decapsulated, weighed, and fixed in $10 \%$ formalin. 
In 10- and 22-day-old groups, India ink injection was performed following clearance and micropuncture experiments reported separately (6). Anesthesia was induced with Inactin, $10 \mathrm{mg} / 100 \mathrm{~g}$ body weight intraperitoneally. A carotid artery and both jugular veins were cannulated with polyethylene tubing for monitoring the arterial blood pressure, infusion of ${ }^{14} \mathrm{C}$ inulin, and donor plasma. The plasma infusion rate was adjusted to maintain a constant hematocrit and blood volume throughout the experiment. The animal was therefore euvolemic at the time of India ink injection, which was performed in a fashion identical to the 1and 105-day-old groups.

The procedure used for glomerular counting was adapted from a modification of the method of Damadian et al. (7) by Kaufman et al. (10). Formalin fixed kidneys were cut into cubes approximately $2 \mathrm{~mm}$ long, placed in $6 \mathrm{ml}$ of $25 \%$ hydrochloric acid, and incubated at $40^{\circ} \mathrm{C}$ for $19-24 \mathrm{~h}$. The time of digestion was found to be critical within $1 / 2 \mathrm{~h}$ : underdigestion resulted in the persistence of aggregated nephrons, and overdigestion led to the presence of numerous glomerular and tubular fragments. Such digests were discarded. Digestion was stopped by the addition of cold distilled water to a total volume of $20 \mathrm{ml}$ and the suspension was mechanically stirred in a $30 \mathrm{ml}$ beaker for $30 \mathrm{~min}$. Aliquots of $25 \mu$ liters were taken at five equally spaced levels in duplicate (total of 10 samples) using an automatic pipette (Eppendorf, Hamburg, W. Germany) and were discharged on microscope slides. For kidneys of 105-day-old animals, $12 \mathrm{ml} \mathrm{HCL}$ was diluted to $40 \mathrm{ml}$, and 50 $\mu \mathrm{l}$ aliquots were used. When the slide was dry, the total number of glomeruli identified by the presence of India ink particles was counted with the aid of a dissecting microscope $(\times 32)$ and grid. Glomerular fragments, half the size of an average glomerulus, were not counted, whereas those larger than this size were included in the total. The number of glomerular fragments was generally less than $5 \%$, and the coefficient of variation averaged $8.6 \%$. The mean number of glomeruli per slide was multiplied by the appropriate dilution factor to obtain the glomerular count per kidney.

Slides originally prepared for glomerular counting were stained with Wright's stain (Camco Quick Stain, Cambridge Chemical Products, Inc., Ft. Lauderdale, FL) to reveal any glomeruli not identified by ink particles. Three slides of different levels were prepared for each of five animals in each of the following groups: 1-day-old, 22-day-old S, 22-day-old N, and 105-day-old S. After
Wright staining, repeat counts of glomeruli containing India ink were not different from those performed before staining, indicating that India ink was not washed off in the process. In order to scan the stained slides closely, the magnification of the dissecting microscope was increased to $\times 64$.

The cortical distribution of glomeruli in seven 22-day-old $\mathrm{N}$ and seven 22-day-old $\mathrm{S}$ groups was investigated by the following procedure. Formalin-fixed, India ink perfused kidneys were bisected longitudinally, and six cores, $3 \mathrm{~mm}$ in diameter, were punched out perpendicular to the surface of the kidney. With the aid of dissecting microscope, medullary tissue was cut away, and the plug of cortical tissue was bisected parallel to the surface, resulting in "outer" and "inner" cortex samples. The six outer cortical samples were pooled, weighed and prepared for glomerular counting and stained as for whole kidneys described above, except that the final volume of the suspension was $10 \mathrm{ml}$. The six inner cortical samples were similarly processed with the final volume of the suspension being $2 \mathrm{ml}$, so that the density of stained glomerular structures was approximately the same for both levels.

The number of glomeruli containing India ink, factored per $\mathrm{g}$ tissue, was then calculated for outer and inner cortex. In five 22day $\mathrm{S}$ animals, the additional glomeruli revealed by Wright's stain were also counted in each cortical level, using the techniques described above for whole kidney counting. The \% of total glomeruli in the outer cortex was calculated for glomeruli containing ink as well as those stained by Wright's stain.

In order to provide histologic correlation of India ink content of glomeruli, four India ink-injected kidneys in each of 1-day-old, 22-day-old S and 22-day-old N groups were fixed in 10\% formalin, embedded in paraffin, sectioned at $10 \mu$, and stained with PAS. Tissue was examined by light microscopy for distribution of ink particles, as well as for the presence of developing glomerular structures.

Results are reported as mean \pm S.E. Linear regression analysis was performed using the method of least squares. Statistical analyses were carried out using Student's $t$ test, with the level of significance being defined as $P<0.05$.

\section{RESULTS}

The mean number of glomeruli identified by India ink in male sham-operated guinea pigs 1-22-days old was $71.1 \pm 2.4 \times 10^{3}$

Table 1. Kidney weight and glomerular number ${ }^{1}$

\begin{tabular}{|c|c|c|c|c|}
\hline \multirow{2}{*}{$\begin{array}{l}\text { Age at } \\
\text { operation }\end{array}$} & \multirow{2}{*}{$\begin{array}{l}\text { Age at study } \\
\text { (days) }\end{array}$} & \multirow{2}{*}{$\begin{array}{l}\text { Kidney wt } \\
\text { (g) }\end{array}$} & \multicolumn{2}{|c|}{$\begin{array}{c}\text { Number of glomeruli per kidney } \\
\text { (thousands) }\end{array}$} \\
\hline & & & India ink & Wright stain \\
\hline & $\begin{array}{l}1.0 \\
(6)\end{array}$ & $\begin{array}{l}0.51 \pm 0.06 \\
\quad(6)\end{array}$ & $\begin{array}{l}71.6 \pm 1.4 \\
(6)\end{array}$ & $\begin{array}{l}9.6 \pm 0.4 \\
(5)\end{array}$ \\
\hline \multicolumn{5}{|l|}{ Sham } \\
\hline Birth & $\begin{array}{l}10.6 \pm 1.0 \\
(5)\end{array}$ & $\begin{array}{l}0.81 \pm 0.06^{2} \\
\quad(5)\end{array}$ & $\begin{array}{l}71.3 \pm 2.2 \\
\quad(5)\end{array}$ & \\
\hline Birth & $\begin{array}{l}22.3 \pm 0.9 \\
\quad(7)\end{array}$ & $\begin{array}{l}1.17 \pm 0.06^{2,3} \\
\quad(7)\end{array}$ & $\begin{array}{l}69.5 \pm 2.5 \\
(7)\end{array}$ & $\begin{array}{l}9.1 \pm 0.9 \\
(5)\end{array}$ \\
\hline 84 days & $\begin{array}{l}106.7 \pm 0.3 \\
\quad(5)\end{array}$ & $\begin{array}{l}2.52 \pm 0.24^{2.3} \\
\quad(5)\end{array}$ & $\begin{array}{l}86.4 \pm 3.5^{2,3} \\
\quad(5)\end{array}$ & $\begin{array}{l}1.4 \pm 0.5^{2,3} \\
(5)\end{array}$ \\
\hline \multicolumn{5}{|c|}{ Uninephrectomy } \\
\hline Birth & $\begin{array}{l}10.5 \pm 0.9 \\
\quad(6)\end{array}$ & $\begin{array}{l}1.24 \pm 0.12^{2,4} \\
\quad(6)\end{array}$ & $\begin{array}{l}75.6 \pm 5.9 \\
(6)\end{array}$ & \\
\hline Birth & $\begin{array}{l}21.6 \pm 0.6 \\
(8)\end{array}$ & $\begin{array}{l}1.80 \pm 0.11^{2.4} \\
\quad(8)\end{array}$ & $\begin{array}{l}83.3 \pm 2.6^{2.4} \\
(8)\end{array}$ & $\begin{array}{l}1.5 \pm 0.4^{2.4} \\
(5)\end{array}$ \\
\hline Birth & $\begin{array}{l}105.0 \pm 0.0 \\
\quad(4)\end{array}$ & $\begin{array}{l}3.46 \pm 0.23^{2} \\
\quad \text { (4) }\end{array}$ & $\begin{array}{l}84.9 \pm 2.7^{2} \\
\quad(4)\end{array}$ & \\
\hline 84 days & $\begin{array}{c}105.3 \pm 0.8 \\
(5)\end{array}$ & $\begin{array}{l}3.29 \pm 0.23^{2} \\
(5)\end{array}$ & $\begin{array}{l}88.2 \pm 2.3^{2} \\
\quad(5)\end{array}$ & \\
\hline
\end{tabular}

\footnotetext{
'Values are mean \pm S.E. (number of animals).

${ }^{2} P<0.01$ versus 1 -day-old group.

${ }^{3} P<0.01$ versus other sham groups.

${ }^{4} P<0.01$ versus same age sham groups.
} 
compared with $69.7 \pm 1.6 \times 10^{3}$ in females of similar age. In 105 day-old animals, the number of glomeruli in males averaged 88.0 $\pm 2.7 \times 10^{3}$, and for females was $87.0 \pm 2.7 \times 10^{3}$. In view of the lack of difference between sexes, males and females were pooled in each of the groups.

As shown in Table 1 and Figure 1 (upper panel) there was no change in glomerular count by India ink perfusion in shamoperated animals during the first 22 days of life, despite a $130 \%$ increase in renal mass. This was in contrast to the ensuing 84 days during which time the number of glomeruli identified by India ink increased by $21 \%$. As illustrated in Table I and Figure 1 (lower panel), in 22-day-old sham-operated animals a mean of 9,140 glomeruli were detected by staining but not by India ink perfusion. This value was not significantly different from that at 1 day of age, and averaged $13 \%$ of the number counted by ink particles alone.

At 10 days of age, animals uninephrectomized at birth manifested a $50 \%$ increase in renal mass compared with the same age $\mathrm{S}$ group, but there was no significant difference in mean number of glomeruli. Twenty-two days following uninephrectomy at brith, the renoprival kidney remained approximately 50\% larger than that of the age-matched sham, but the number of glomeruli identified by India ink was $20 \%$ greater. By 105 days of age, there was no further increase in glomerular number in this group. Unilateral nephrectomy at 84 days of age resulted in only a $30 \%$ compensatory increase in renal mass 21 days later and there was no effect on glomerular number. There were significantly fewer glomeruli identified by staining in the 22-day-old uninephrectomized animals, with a mean of 1,500 glomeruli, or $2 \%$ of the number identified by ink. This result was not different from that of the adult sham-operated guinea pigs, with an average of 1,400 glomeruli free of identifiable India ink.

The distribution of glomeruli in outer and inner cortex of 22day-old animals is shown in Table 2. The concentration of glomeruli identified by ink in outer cortex is significantly greater in $\mathrm{S}$ than $\mathrm{N}$ animals. The difference in inner cortex is of borderline

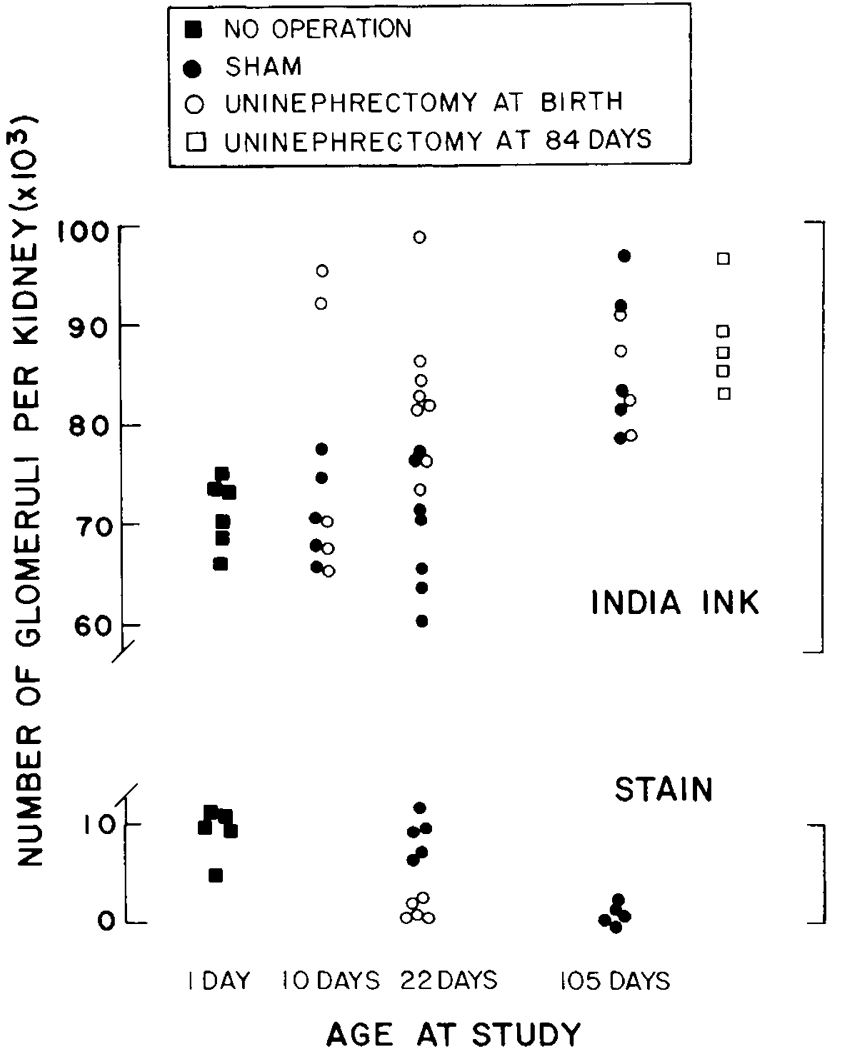

Fig. 1. Number of glomeruli identified by India ink (upper panel) and by Wright's stain (lower panel). Each point represents one animal.
Table 2. Cortical distribution of glomeruli in 22-day-old guinea

\begin{tabular}{|c|c|c|c|c|}
\hline & $\begin{array}{l}\text { Uninephrectomy } \\
\text { India ink } \\
\text { (7) }\end{array}$ & & $\begin{array}{l}\text { Sham } \\
\text { India } \\
\text { ink } \\
(7)\end{array}$ & $\begin{array}{l}\text { Sham } \\
\text { Wright } \\
\text { stain } \\
(5)\end{array}$ \\
\hline $\mathrm{OC}^{2} \times 10^{3} / \mathrm{g}$ & $89 \pm 6$ & $P<0.01$ & $137 \pm 12$ & $23 \pm 3$ \\
\hline $\mathrm{IC}^{2} \times 10^{3} / \mathrm{g}$ & $30 \pm 3$ & $P=0.05$ & $39 \pm 4$ & $7 \pm 1$ \\
\hline$\frac{\mathrm{OC}}{\mathrm{OC}+\mathrm{IC}} \%$ & $75 \pm 2$ & $P=0.45$ & $77 \pm 2$ & $77 \pm 2$ \\
\hline
\end{tabular}

${ }^{1}$ Values are mean \pm S.E. (number of animals).

${ }^{2} \mathrm{OC}$, outer cortex; IC, inner cortex.

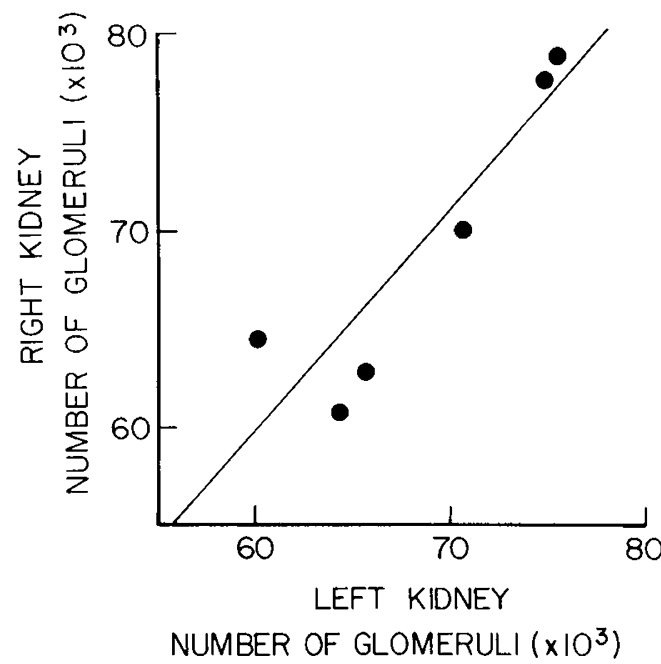

Fig. 2. Number of glomeruli identified by India ink: relationship between right and left kidneys $(\mathrm{y}=1.5 \mathrm{x}-9.25, r=0.92, P<0.01)$.

significance. The $\%$ of total glomeruli in the outer cortex was not different in the two groups; however, in the S group, the outer cortical distribution of glomeruli identified by Wright's stain was similar at $77 \%$. The ratio of stained glomeruli to those containing ink in this group was $17 \%$ in both outer and inner cortex.

Figure 2 shows the significant relationship between left and right kidneys for glomerular number determined by India ink in six animals ranging in age from 1-49 days. There was also a significant correlation between left and right kidney weight of individual animals $(r=0.999, P<0.001)$. When linear regression analysis was applied to the left kidney weight versus glomerular number for all 1-22-day-old animals in the study, no correlation was demonstrated: $r=0.16, P=\mathrm{NS}(n=17)$.

Paraffin sections of kidneys perfused by India ink revealed scattered glomeruli not containing ink in 1-day-old (Fig. 3) and 22-day-old $\mathrm{S}$ guinea pigs, but almost none in 22-day-old $\mathrm{N}$ animals. Although most of the ink-free glomeruli were located in the outer cortex, they were also identified in the juxtamedullary region. The ink-free glomeruli appeared to have fewer capillary loops than those containing ink particles. There were no glomeruli in an early stage of formation (i.e., S-shaped bodies or renal vesicles) in any of the sections from 1-day-old or 22-day-old $S$ or $\mathrm{N}$ guinea pigs.

\section{DISCUSSION}

In the present study, the number of glomeruli per kidney identified by India ink perfusion increased approximately $20 \%$ from birth to adulthood. A significant and quantitatively similar increase was also reported by Bonvalet et al. (3) for guinea pigs 120 days of age compared with 90 -day-old animals; and by MerletBenichou and de Rouffignac (13) for guinea pigs in the first 6 wk 
of life. In another study (16), no increase was found from 12-49 days of age, but only three animals were examined, and the number of glomeruli was approximately half that reported elsewhere. It is possible that strain differences or over-digestion of renal tissue may account for this discrepancy.

The discovery in 1-22-day-old guinea pigs of additional glomeruli not containing India ink particles, suggests that a number of previous developmental studies of glomerular number must be interpreted with caution. In their original report, Damadian et al. (7) did not intend the technique to be used as a measure of total glomerular number, but rather to identify those with "patent, perfused glomerular capillaries." Consistent with the present study, Kunkel (11) found that (2-10\%) of glomeruli in the developing guinea pig were uninjected by Prussian blue used as a glomerular marker. It is not stated, however, whether the highest $\%$ of uninjected glomeruli was present in the youngest animals.

A new and unexpected finding in the present study was the $20 \%$ increase in number of glomeruli detected by India ink of 22-dayold renoprival guinea pigs compared with age-matched shams. The discrepancy between the increase in number of glomeruli identified by India ink for 22-day-old $\mathrm{N}$ compared with $\mathrm{S}$ groups $(20 \%)$ and the additional stained glomeruli in the 22 day $S$ group (13\%) may be due to obscuring of some ink-free glomeruli by overlapping tubular structures which also become stained by Wright's stain. This is supported by the higher \% of stained glomeruli $(17 \%)$ in the more dilute preparation of cortical tissue. In view of the lack of developing glomerular structures in histologic sections, it is therefore likely that the total number of nephrons does not change after birth, even following reduction of renal mass, and that the increase in number of glomeruli identified by India ink reflects enhanced perfusion of $13-20 \%$ of the glomeruli.

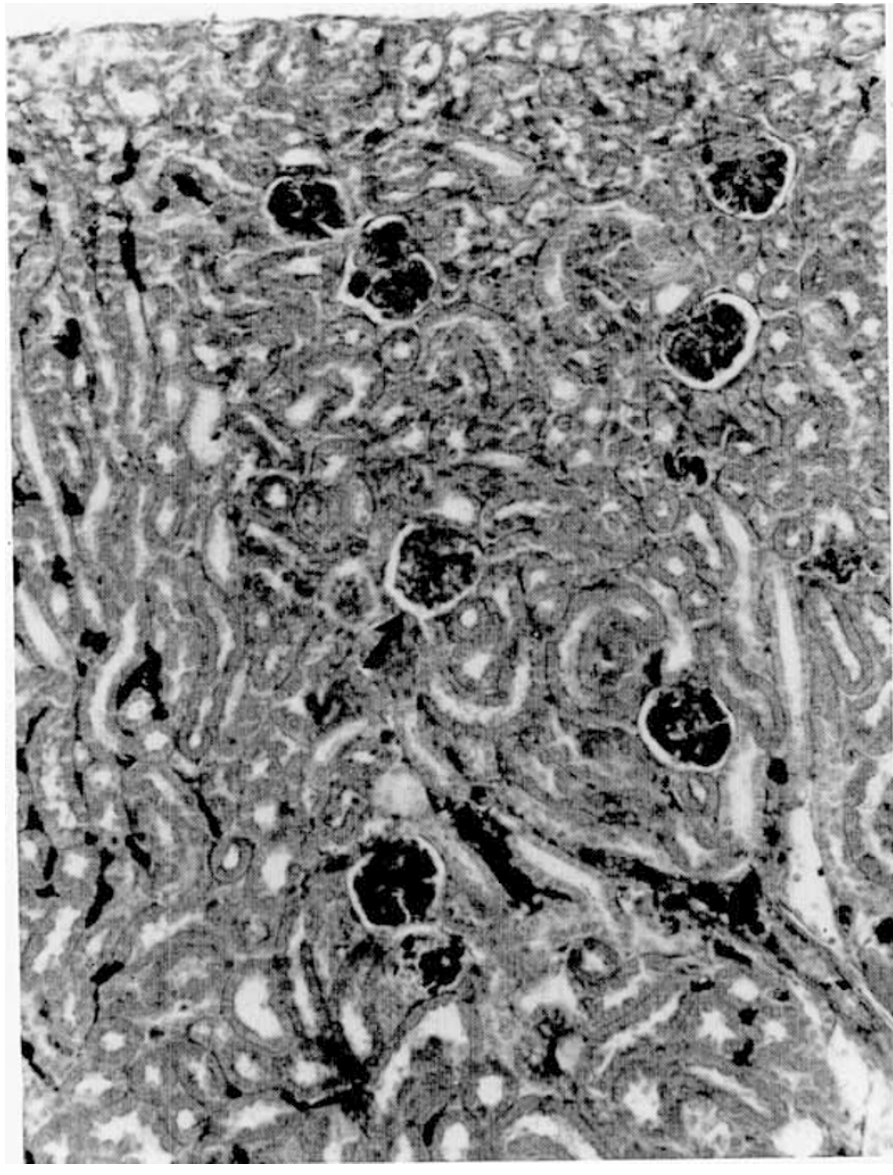

Fig. 3. India ink-perfused kidney from 1-day-old guinea pig. Arrow indicates glomerulus lacking ink, surrounded by glomeruli containing ink particles $(\times 125)$.
In a recent study of the uninephrectomized newborn rat (12), histologic evidence of nephron formation was also lacking, and glomerular number was not altered at 60 days of age. These authors postulate that the apparent increased number of glomeruli previously reported in renoprival developing rats may be due to accelerated development of preexisting nephrons (12). The inverse correlation in the present study of ink-containing and ink-lacking glomeruli supports this contention for the guinea pig as well, and provides an explanation for the lower number of glomeruli identified by India ink in early development. Another possible artifact of the method of Damadian et al. is over-digestion of immature glomeruli in the neonatal kidney due to their increased fragility (12). Although representing a potential problem in digestion of fresh tissue $(3,13)$, formalin fixation of kidneys in the present study would minimize this effect.

The decrease in concentration of glomeruli throughout the cortex as a result of uninephrectomy is most likely due to the compensatory increase in tubular mass. Because $3 / 4$ of all glomeruli identified by India ink and Wright stain are present in the outer cortex of 22-day-old S animals, and the distribution of glomeruli containing ink is the same in uninephrectomized animals, it follows that there was a 3 -fold greater recruitment of superficial compared to deep nephrons at this age. It is unlikely that a rapid increase in total renal blood flow to the remaining kidney after uninephrectomy could explain the changes because there is no significant difference in number of glomeruli identified by India ink in 10-day-old $\mathrm{N}$ and $\mathrm{S}$ groups. As shown in Figure 1, however, two uninephrectomized guinea pigs had more than 90,000 glomeruli by 10 days, presumably due to interanimal variability in timing of glomerular recruitment. A simple relationship between renal mass and glomeruli containing ink is improbable because there is no increase in number of glomeruli during the first $3 \mathrm{wk}$ of life despite a doubling of kidney weight. It is possible that altered glomerular perfusion in the developing guinea pig may not take place until 3-5 wk of age, similar to the delayed increase in outer cortical superficial nephron glomerular filtration rate (16).

Determination of glomerular number in kidneys of various species has been accomplished by two basic techniques: counting all glomeruli in serial sections, a tedious procedure which limits the number of animals studied; and counting glomeruli in aliquots of acid digests of kidneys perfused with a marker such as Prussian blue (11), India ink (7), or even residual blood (5). Although serial sections were utilized in the earliest studies $(1,2)$, the acid digest method was established over 50 years ago (11) and gained renewed interest with the use of India ink as a marker of glomerular perfusion by Damadian et al. (7).

In the present study, a close correlation was found between number of glomeruli identified by India ink in the right and left kidneys: the difference averaged $4 \pm 1 \%(n=6)$ (Fig. 2) compared with a mean spread of $25 \pm 4 \%(n=8)$ for number of glomeruli per left kidney within each group. This would suggest that in the guinea pig, the number of functioning glomeruli is synchronized for both kidneys during early development, and that the greater variability between individual animals is not due to lack of precision in technique. Damadian et al. (7) also reported a close correlation between right and left glomerular counts in the dog, with $r=0.98, P<0.01(n=5)$. This was not found by Kaufman et al. (9) in the rat, however, as linear regression analysis reveals $r=0.22, P=$ not significant $(n=10)$. It is not clear whether such variability is due to methodologic or species differences.

Because all animals underwent unilateral carotid artery cannulation, this procedure would not be expected to affect the observed differences in renal perfusion. The physiologic state of the animal at the time of study was likewise controlled. One-dayold and adult 105-day-old guinea pigs were treated identically and were not subjected to dehydration. In 10- and 22-day-old $\mathrm{N}$ and $\mathrm{S}$ groups, arterial blood pressure and glomerular filtration rate were constant, and euvolemia was maintained (6). One possibility may be the presence of vascular communications between interlobular artery and vein, that have been described in early renal development (14). Alternatively, there may be variable focal vas- 
cular reactivity throughout the developing kidney, with an acceleration of the mature pattern resulting from neonatal uninephrectomy. The functional correlates of accelerated recruitment of additional nephrons in 22-day-old guinea pigs uninephrectomized at birth are the subject of a separate study (6). It was found that compensatory increase in single nephron glomerular filtration rate at that age is proportionately less than the increase in whole kidney filtration rate, and the difference could be explained by the contribution of recruited glomeruli. Such changes may play an important role in the early, nearly complete, compensatory adaptation of the guinea pig subjected to uninephrectomy at birth.

\section{REFERENCES AND NOTES}

1. Arataki, M.: On the postnatal growth of the kidney, with special reference to the number and size of the glomeruli (albino rat). Am. J. Anat., 36: 399 (1926).

2. Arataki, M.: Experimental researches on the compensatory enlargement of the surviving kidney after unilateral nephrectomy (albino rat). Am. J. Anat., 36: 437 (1926).

3. Bonvalet, J. P., Champion, M., Courtalon, A., Farman, N., Vandewalle, A., and Wanstok, F.: Number of glomeruli in normal and hypertrophied kidneys of mice and guinea-pigs. J. Physiol. (Lond), 269: 627. (1977).

4. Bonvalet, J. P., Champion, M., Wanstok, F., and Berjal, G.: Compensatory renal hypertrophy in young rats: increase in the number of nephrons. Kidney Intern., I: $391(1972)$.

5. Canter, C. E. and Goss, R. J.: Induction of extra nephrons in unilaterally nephrectomized immature rats. Proc. Soc. Exp. Bio. Med., 148: 294 (1975).

6. Chevalier, R. L.: Functional adaptation to reduced renal mass in early development. Am. J. Physiol,, 242: F190 (1982).

7. Damadian, R. V., Shwayri, E., and Bricker, N. S.: On the existence of non-urine forming nephrons in the diseased kidney of the dog. J. Lab. Clin. Med., 65: 26 (1965).
8. Jackson, C. M. and Shiels, M.: Compensatory hypertrophy of the kidney during various periods after unilateral nephrectomy in very young albino rats. Anat. Rec., 36: 221 (1927)

9. Kaufman, J. M., DiMeola, H. J., Siegel, N. J., Lytton, B., Kashgarian, M., and Hayslett, J. P.: Compensatory adaptation of structure and function following progressive renal ablation. Kidney Intern., 6: 10 (1974).

10. Kaufman, J. M., Hardy, R., and Hayslett, J. P.: Age-dependent characteristics of compensatory renal growth. Kidney Intern., 8: 21 (1975).

11. Kunkel, P. A. Jr., : The number and size of the glomeruli in the kidney of several mammals. Bull. Johns Hopkins Hosp., XLVII: 285 (1930).

12. Larsson L., Aperia, A., and Wilton, P.: Effect of normal development on compensatory renal growth. Kidney Intern., 18: 29 (1980).

13. Merlet-Benichou, C. and de Rouffignac, C.: Renal clearance studies in fetal and young guinea pigs: effect of salt loading. Am. J. Physiol., 232: F178 1977).

14. Potter, E. L.: Normal and Abnormal Development of the Kidney. p. 59 (Chicago, Year Book Medical Publishers, Inc., Chicago, IL 1972).

15. Solomon, S.: Developmental changes in nephron number, proximal tubular length and superficial nephron glomerular filtration rate of rats. J. Physiol. (Lond), 272: 573, (1977).

16. Spitzer, A. and Brandis, M.: Functional and morphologic maturation of the superficial nephrons: relationship to total kidney function. J. Clin. Invest., 53: 279 (1974)

17. This study was presented in part at the annual meeting of the Society for Pediatric Research, San Francisco, California, April 29, 1981.

18. The author is indebted to James G. Bowman, Jr., for his excellent technical assistance, and the helpful suggestions of Drs. Carl W. Gottschalk and Stuart S. Howards are greatfully acknowledged.

19. Requests for reprints should be addressed to: Robert L. Chevalier, M. D. Department of Pediatrics, Box 386, University of Virginia Medical School, Charlottesville, Virginia 22908. U.S.A.

20. This research was supported by the National Institutes of Health Grant R01-AM 25727; by Grant-in-Aid 79-614 from the American Heart Association, Virginia Affiliate; and by Biomedical Research Support Grant 5-207-RR05431-17 from the National Institutes of Health.

21. Received for publication June 15, 1981.

22. Accepted for publication September 25, 1981. 